\title{
A Rare Case of Mesothelioma Showing Micropapillary and Small Cell Differentiation with Aggressive Behavior
}

\author{
Yoon Jin Cha $\cdot$ Binnari Kim $\cdot$ Joungho Han $\cdot$ Chin A Yi ${ }^{1} \cdot$ Jae Ill Zo ${ }^{2}$ \\ Departments of Pathology, ${ }^{1}$ Radiology, and ${ }^{2}$ Thoracic Surgery, Samsung Medical Center, Sungkyunkwan University School of Medicine, Seoul, Korea
}

Pleural mesothelioma is a rare neoplasm and roughly $70 \%$ are the epithelioid type, which is characterized by proliferation of epithelioid tumor cells with a tubulopapillary growth pattern. ${ }^{1}$ There have been few reports of mesothelioma having a micropapillary pattern ${ }^{2}$ and a small cell component. ${ }^{3}$ To the best of our knowledge, there has been no previously reported case containing both micropapillary and small cell components. Herein, we report a rare case of mesothelioma with micropapillary component and small cell differentiation exhibiting aggressive behavior in a 59-year-old woman.

\section{CASE REPORT}

A 59-year-old female presented with a 2-month history of pleuritic chest pain aggravated by exercise and breathing. She had hyperthyroidism and had been on medication for 10 years, but had no history of occupational or environmental exposure to asbestos. A computed tomography (CT) examination revealed band-like thickening along the right pleura and fissure, suggesting malignant mesothelioma. After video-assisted thoracoscopic surgery of the right pleura, she diagnosed with malignant mesothelioma, epithelioid type. Despite of neoadjuvant chemotherapy, right pleural and fissural thickening was found to have progressed on CT (Fig. 1A). The patient underwent right extrapleural pneumonectomy.

The right lung was entirely encased with diffusely thickened

\section{Corresponding Author}

Joungho Han, M.D.

Department of Pathology, Samsung Medical Center, Sungkyunkwan University School of Medicine, 81 Irwon-ro, Gangnam-gu, Seoul 135-710, Korea

Tel: +82-2-3410-2800, Fax: +82-2-3410-0025, E-mail: hanjho@skku.edu

Received: July 1, 2014 Revised: July 30, 2014

Accepted: August 1, 2014 pleura (Fig. 1B). The tumor involved the visceral pleura, infiltrated the parietal pleura and lung parenchyma, and was accompanied by thick fibrosis. Discohesive and epithelioid tumor cells were arranged in a solid sheet and partly micropapillary patterns. Marked nuclear pleomorphism and frequent mitoses were found. The tumor cells frequently invaded lymphatic spaces and metastasized to 35 lymph nodes. There were two distinct subpopulations of tumor cells: micropapillary and small cell components (Fig. 1C-E). The micropapillary component at invasive fronts (Fig. 1C) had no fibrovascular core and was reminiscent of micropapillary adenocarcinoma of the lungs (Fig. 1D). The small cell component was found in multifocal areas of the tumor, admixed with larger epithelioid cells arranged in micropapillary pattern (Fig. 1E). This component resembled small cell carcinoma of the lung, having a high nuclear-cytoplasmic ratio, and a finely dispersed "salt-and-pepper" chromatin pattern. Ultrastructural study of the tumor revealed long, slender microvilli on the cell surface, which supported the diagnosis of mesothelioma (Fig. 1F). Immunohistochemical staining for calretinin, D2-40, thyroid transcription factor-1 (TTF-1), Wilms' tumor-1 (WT-1), HBME-1, CD56, and p16 were performed. TTF-1 was totally negative in the tumor. Calretinin expression in the micropapillary component was weak and patchy, and the small cell component showed only a few positive cells (Fig. 1G). D2-40 (Fig. 1H) and HBME-1 (Fig. 1I) delineated the tumor cell membrane, which is a distinct and useful feature in the diagnosis of mesothelioma. WT-1 showed diffuse nuclear expression in both micropapillary and small cell components (Fig. 1J). CD56 exhibited membranous positivity in the small cell component (Fig. 1K). p16 showed diffuse and strong nuclear and cytoplasmic expression in the tumor cells (Fig. 1L). 

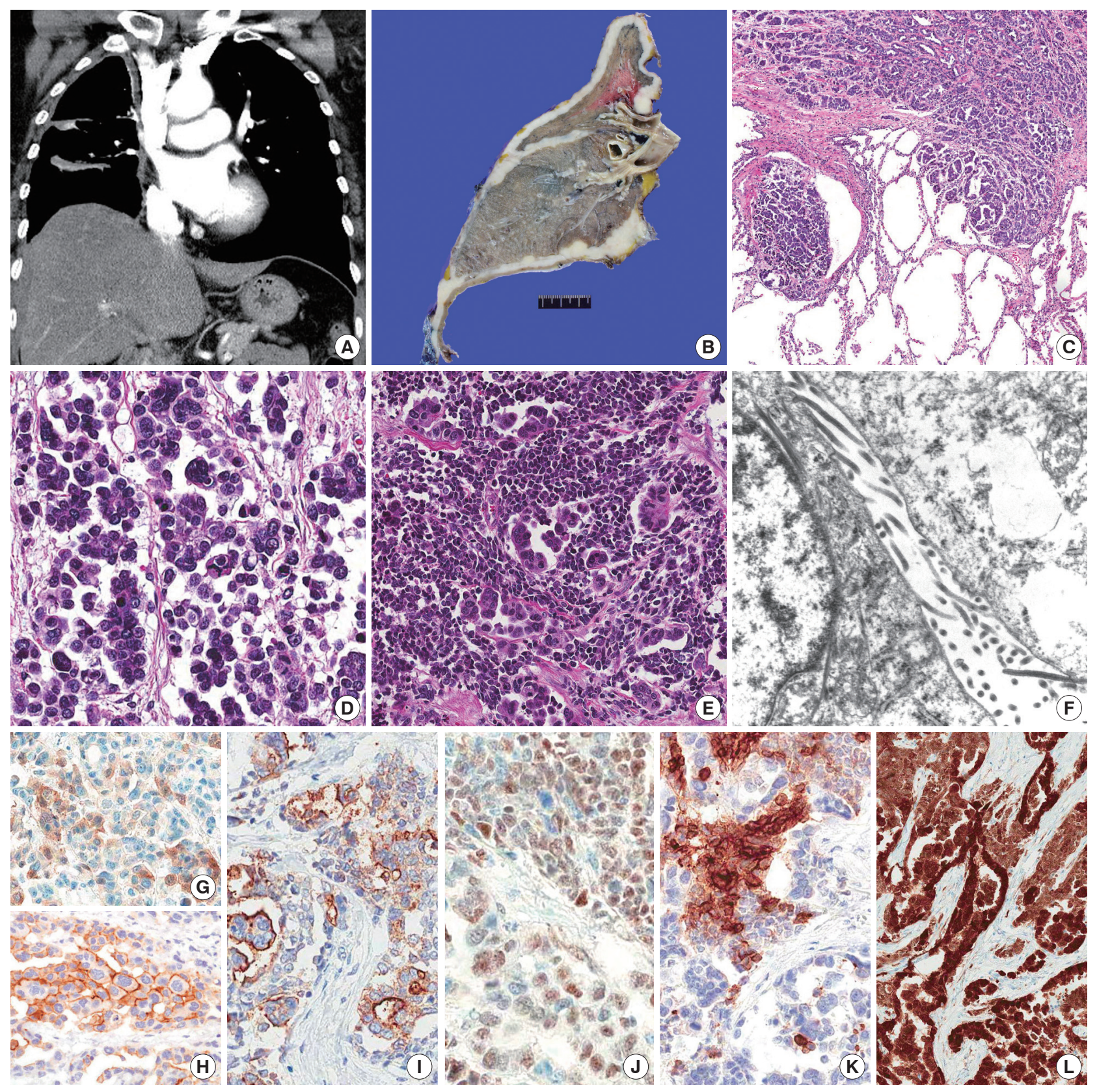

Fig. 1. (A, B) Computed tomography image and gross findings show diffusely thickened mediastinal and fissural pleura. (C) The micropapillary component shows lymphatic invasion and infiltration into lung parenchyma. (D) The micropapillary component has no fibrovascular core, reminiscent of micropapillary adenocarcinoma of the lung. (E) The small cell component shows a high nuclear-cytoplasmic ratio and a sheetlike arrangement compared to admixed micropapillary component. (F) Ultarstructural finding shows long, slender microvilli on the tumor cell surface. (G) Calretinin shows patchy staining of nuclei and cytoplasm. (H) D2-40 is positive on the membrane of tumor cells. (I) HBME-1 is positive on the membrane of tumor cells. (J) WT-1 shows diffuse nuclear positivity. (K) The small cell component shows membranous expression of CD56. (L) p16 is diffuse and strongly positive throughout the tumor.

\section{DISCUSSION}

Mesothelial cells can show variable architecture and cytological atypia, and thus, distinguishing benign mesothelial hyperplasia, malignant mesothelioma, metastatic adenocarcinoma from lung, and rarely malignant melanoma can be difficult. An immunohistochemical panel including calretinin, WT-1, HBME1, TTF-1, carcinoembryonic antigen (CEA), and MOC-31 helps to differentiate metastatic lung adenocarcinoma and mesothelioma. ${ }^{4}$ 
In the present case, the tumor lacked TTF-1 expression and was only focally positive for calretinin, which did not fit the typical diagnosis of mesothelioma or lung adenocarcinoma well. However, clinical, radiological, histological, and ultrastructural features along with D2-40, HBME-1, and WT-1 expression were definitive for a diagnosis of mesothelioma. A micropapillary pattern in mesothelioma has been rarely reported, ${ }^{2}$ but, when present, this growth pattern shows poor prognosis with aggressive lymphatic invasion and lymph node metastasis. In a previous study of small cell mesothelioma, tumor cells showed immunoreactivity for neuron-specific enolase and cytokeratin, but not for chromogranin A and CEA. ${ }^{3}$ Rarely, pulmonary and extrapulmonary small cell carcinoma mimics mesothelioma. ${ }^{5}$ As a tumor suppressor, inactivation of $\mathrm{p} 16$ by homozygous deletion, promoter methylation, and point mutation has been demonstrated in carcinogenesis. A recent study has shown that deletion of p16/CDKN2A is occurs in mesothelioma, but not in benign mesothelial cells, which can be detected by fluorescence in situ hybridization. ${ }^{6}$ Aberrant expression of p16 on immunohistochemical staining has been reported in breast and colorectal carcinoma, which exhibit p16 inactivation and poor prognosis. ${ }^{7}$

We report a mesothelioma with uncommon growth patterns - micropapillary and CD56-expressing small cells-which showed rapid progression despite chemotherapy, aggressive lymphatic invasion and lymph node metastasis.

\section{Conflicts of Interest}

No potential conflict of interest relevant to this article was reported.

\section{Acknowledgments}

This study was partly supported by the Korean Ministry of the Environment "Environmental Health Action Program." We are grateful to Dr. Kun Young Kwon of Keimyung University Dongsan Medical Center who performed the ultrastructral study and helpful advice.

\section{REFERENCES}

1. Travis WD, Brambilla E, Muller-Hermelink HK, Harris CC. Pathology and genetics of tumours of the lung, pleura, thymus and heart. Lyon: IARC Press, 2004.

2. Mogi A, Nabeshima K, Hamasaki M, et al. Pleural malignant mesothelioma with invasive micropapillary component and its association with pulmonary metastasis. Pathol Int 2009; 59: 874-9.

3. Mayall FG, Gibbs AR. The histology and immunohistochemistry of small cell mesothelioma. Histopathology 1992; 20: 47-51.

4. Ahn S, Choi IH, Han J, Kim J, Ahn MJ. Pleural mesothelioma: an institutional experience of 66 cases. Korean J Pathol 2014; 48: 91-9.

5. Noguchi K, Fujimoto N, Asano M, et al. Extrapulmonary small cell carcinoma mimicking malignant pleural mesothelioma. J Clin Pathol 2013; 66: 450-1.

6. Chung CT, Santos Gda C, Hwang DM, et al. FISH assay development for the detection of p16/CDKN2A deletion in malignant pleural mesothelioma. J Clin Pathol 2010; 63: 630-4.

7. Cui X, Shirai Y, Wakai T, Yokoyama N, Hirano S, Hatakeyama K. Aberrant expression of $\mathrm{pRb}$ and $\mathrm{p} 16(\mathrm{INK} 4)$, alone or in combination, indicates poor outcome after resection in patients with colorectal carcinoma. Hum Pathol 2004; 35: 1189-95. 